INPLASY

PROTOCOL

To cite: Li et al. Diagnostic accuracy of synovial fluid Dlactate for periprosthetic joint infection: a systematic review and meta-analysis. Inplasy protocol 202190036. doi: 10.37766/inplasy2021.9.0036

Received: 13 September 2021

Published: 13 September 2021

Corresponding author:

Wei Sun

cjfhsunw@163.com

Author Affiliation: China-Japan friendship hospital

Support: None.

Review Stage at time of this submission: Preliminary searches.

Conflicts of interest: None declared.

\section{Diagnostic accuracy of synovial fluid D-lactate for periprosthetic joint infection: a systematic review and meta-analysis}

Li, Z1; Li, C2; Wang, G3; Li, T4; Fan, X5; Xu, X6; Wang, P7; Sun, W8.

Review question / Objective: Synovial fluid D-lactate were proposed as novel diagnostic biomarkers for PJI by Yermak et al. in 2019, and it has also been reported with high sensitivity and specificity. But the clinical value of D-lactate in assessing PJI is still under debate and investigation. The aim of this study systematic review and meta-analysis was to synthesize the published data on the accuracy of D-lactate in the detection of PJI, and assess the diagnostic value of D-lactate for PJI.

Condition being studied: Arthroplasty is an effective treatment of end-stage joint diseases. With the continuous improvement of the surgery, the complications are gradually reduced, but it is still unavoidable, in which periprosthetic joint infection (PJI) is the most serious complications. Prompt establishment of an infection diagnosis is critical but can be very challenging at present.

INPLASY registration number: This protocol was registered with the International Platform of Registered Systematic Review and Meta-Analysis Protocols (INPLASY) on 13 September 2021 and was last updated on 13 September 2021 (registration number INPLASY202190036).

\section{INTRODUCTION}

Review question / Objective: Synovial fluid D-lactate were proposed as novel diagnostic biomarkers for PJI by Yermak et al. in 2019, and it has also been reported with high sensitivity and specificity. But the clinical value of D-lactate in assessing PJI is still under debate and investigation. The aim of this study systematic review and meta-analysis was to synthesize the published data on the accuracy of D- 
lactate in the detection of PJI, and assess the diagnostic value of D-lactate for PJI.

Condition being studied: Arthroplasty is an effective treatment of end-stage joint diseases. With the continuous improvement of the surgery, the complications are gradually reduced, but it is still unavoidable, in which periprosthetic joint infection (PJI) is the most serious complications. Prompt establishment of an infection diagnosis is critical but can be very challenging at present.

\section{METHODS}

Participant or population: Population, patients who had undergone arthroplasty.

Intervention: Using D-lactate test to diagnose PJI.

Comparator: Diagnostic gold standard MSIS OR EBJIS.

Study designs to be included: Retrospective and prospective studies.

Eligibility criteria: (1) population, patients who had undergone arthroplasty, (2) performed the D-lactate test in synovial fluid, (3) the diagnosis of PJI was confirmed by the MSIS, AAOS or EBJIS guidelines and (4) sufficient data could be extracted for constructing a $2 \times 2$ contingency table. Case reports, commentaries, expert opinion, narrative reviews and studies carried out in animals were excluded.

Information sources: We will search three electronic databases including PubMed, Embase, and Cochrane Library without time limitation. Vocabulary and syntax were specifically adapted according to the database. We used "periprosthetic joint infection" or "prosthesis related infections" as our diagnosis of interest and "lactate" as our target index. No language limitation was applied. Reference lists of relevant articles were also screened manually for any additionally possible records.
Main outcome(s): The pooled sensitivity, pooled specificity, pooled likelihood ratios, diagnostic odds ratio (DOR).

Quality assessment / Risk of bias analysis: Methodological quality of included studies was appraised according the QUADAS (Quality Assessment of Diagnostic Accuracy Studies)-2 tool, which consists of four key domains (i.e., patient selection, index test, reference standard, and flow and timing). Risk of bias was assessed in each domain and concerns about applicability were assessed in the first three domains with signaling questions.

Strategy of data synthesis: Heterogeneity among included studies was assessed using the 12 statistic. An 12 value of $0 \%$ implied no observed heterogeneity, and values of $>50 \%$ indicated substantial heterogeneity. We also performed Deek's funnel plot asymmetry test to determine if there is publication bias.

Subgroup analysis: None.

Sensitivity analysis: None.

Country(ies) involved: China.

Keywords: Periprosthetic joint infection; D -lactate; Arthroplasty; Meta-Analysis; Synovial fluid.

Contributions of each author:

Author 1 - Zhizhuo Li.

Author 2 - Chengxin Li.

Author 3 - Guangxue Wang.

Email: 1810122717@pku.edu.cn

Author 4 - Tengqi Li.

Author 5 - Xiaoyu Fan.

Author 6 - Xin Xu.

Author 7 - Peixu Wang.

Author 8 - Wei Sun.

Email: cjfhsunw@163.com 